\title{
A Systematic Study of Cubic Equations of State with van der Waals Mixing Rules and Different Combining Rules in Predicting the Densities of LNG and Natural Gas Model Systems
}

\author{
M. S. Al-Manthari, M. A. Al-Wadhahi, G. Reza Vakili-Nezhaad*, K. Nasrifar \\ Petroleum and Chemical Engineering Department, Sultan Qaboos University, Muscat, Oman \\ E-mail: *alwadahi@squ.edu.om
}

Received 17 February 2019, Revised 21 April 2019, Accepted 07 May 2019

\begin{abstract}
Cubic equations (EoSs) of state are successfully used in petroleum and natural gas industry. In order to extend these equations to mixtures, van der Waals mixing rules with Lorentz-Berthelot (LB) combining rules are often employed; however, the accuracies of these EoSs in predicting the liquid densities of hydrocarbon mixtures are not adequate. The main objective of this study was comparing 13 EoSs coupled with 10 combining rules in predicting the densities of hydrocarbon mixtures. Binary and ternary liquid mixtures, LNG mixtures and synthetic natural gas mixtures comprising 752 data points were collected and used in this study. Results revealed that for predicting the liquid densities of binary and ternary mixtures, the Schmidt and Wenzel (SW) EoS coupled with Hudson-McCoubrey (HMC) or LB combining rules are the best among the others. The SW EoS coupled with the LB combining rules were also the best in predicting the densities of the LNG mixtures. Additionally, the LB combining rules are the best in predicting natural gas mixtures densities using the Patel and Teja (PT), SW and Patel-Teja-Valderrama (PTV) EoSs. In general, it was found that the Redlich-Kwong (RK) family EoSs, SW and Trebble-Bishnoi-Salim (TBS) EoSs are best coupled with the LB combining rules. However, the Peng and Robinson (PR) family EoSs with Halgren (HHG) combining rules were in better agreement with experimental data. The Waldman-Hagler (WH) combining rules lacked predictability when coupled with the most EoSs.
\end{abstract}

Keywords: Density; natural gas, hydrocarbon, equation of state, combining rules.

\section{Introduction}

In natural gas and petroleum engineering, cubic equations of state (EoSs) are important in equilibrium calculations. Often the accuracy of the EoSs in equilibrium calculations are not identical and comparisons are made to find the best EoS among the others. For instance, Nasrifar et al. [1] compared 15 EoSs in predicting lean and rich natural gas dew points. They found that the dew points of lean synthetic natural gases are predicted best by the RedlichKwong (RK) family EoSs [2] and the Schmidt and Wenzel (SW) EoS [3] whereas the three-parameter EoSs like PatelTeja (PT) [4], Patel-Teja-Valderrama (PTV) [5] and Guo-Du (GD) $[6,7]$ are the best in predicting the dew points of rich natural gases.

Equally important is the application of EoSs in predicting the pressure-volume-temperature (PVT) and the thermodynamic properties of hydrocarbon fluids. Coats and Smarts [8] conducted a study where the Peng-Robinson (PR) [9] and Zudkevitch-Joffe-Redlich-Kwong (ZJRK) EoSs [10] were applied to correlate and predict fluid phase properties. The results showed that the agreement between laboratory properties, such as gas oil ratio and stock tank oil gravity, and the EoS calculated values was generally good to excellent. However, both EoSs gave generally poor agreement with any set of laboratory PVT data. Ahmed [11] compared eight cubic EoSs in predicting the volumetric and phase equilibria of gas condensate systems. He found that the SW EoS exhibits a superior predictive capability for volumetric properties of gas-condensate systems. The PT and SW EoSs were found to give reliable gas density predictions and in terms of vapor-liquid equilibrium (VLE) calculations, the PR, PT, and SW EoSs all performed equally well. Nasrifar and Bolland [12] compared 10 EoSs including a proposed EoS to predict thermodynamic properties such as saturated liquid density of LNG mixtures, compressibility factors, speeds of sound, isobaric heat capacity and JouleThomson coefficient of natural gas mixtures. Their results indicated that the saturated liquid density of the LNG mixtures was predicted best by the modified NasrifarMoshfeghian (MNM) [13] and Mohsennia-ModarressMansoori (3M) EoSs [14] and the accuracy of the proposed EoS was between the Redlich-Kwong-Soave (RKS) [15] and PR family EoSs. For predicting the compressibility factor and speeds of sound of natural gas mixtures, the proposed EoS was found to be the most accurate EoS among the others; however, the accuracy of the proposed EoS in predicting the isobaric heat capacity and Joule-Thomson coefficient was found comparable to the RKS EoS or one of its variants.

EoSs are in plenty. The PVT of an EoS accounts for its accuracy and exact thermodynamic relations are often expressed in terms of the PVT of fluids [16]. Accurate PVT relation for an EoS then leads to accurate thermodynamic property calculation. For instance, the RK EoS or one of its variants describes well the PVT of natural gas mixtures. For hydrocarbon mixtures with heavy fractions, however, the PR EoS is somewhat more accurate, especially, in predicting 
liquid density. These EoSs are still subject of research to improve their accuracies $[17,18]$.

Dealing with mixtures, mixing rules also play a vital role. For hydrocarbon mixtures, van der Waals mixing rules are invariably used in equilibrium calculations [19].

Lennard-Jones potential is frequently used to describe the interaction between molecules. Because of its simplicity, it has been applied in many molecular simulations for thermodynamic properties of simple fluids. This model can be expressed by:

$u_{L J}=4 \varepsilon\left[\left(\frac{\sigma}{r}\right)^{12}-\left(\frac{\sigma}{r}\right)^{6}\right]$

where $\varepsilon$ and $\sigma$ are the energy and size parameters and $r$ is the distance between the molecules. For unlike pairs of molecules we need to introduce proper combining rules for size and energy parameters. One of the oldest and the most used combing rules for these parameters have been proposed by Lorentz [20] and Berthelot [21]. Lorentz combining rule for size parameter $(\sigma)$ which is correct for hard sphere potential models is an arithmetic average of the size parameters of two unlike molecules read as

$\sigma_{i j}=\frac{\sigma_{i}+\sigma_{j}}{2}$

For the energy parameter, the geometric average of two unlike molecules has been proposed by Berthelot as follows

$\varepsilon_{i j}=\sqrt{\varepsilon_{i} \varepsilon_{j}}$

These two equations known as Lorentz-Berthelot (LB) combining rules have been widely used in molecular dynamics simulations. Based on LB combining rules, almost all cubic equations of state followed the same rules for calculation of the energy (aij) and size parameter (bij) for unlike pairs of components in a multicomponent mixture, read as

$a_{i j}=\sqrt{a_{i} a_{j}}$

$b_{i j}=\frac{b_{i}+b_{j}}{2}$

In spite of successful application of the above mentioned rules for the volumetric and phase equilibrium calculations for multicomponent mixtures, serious deficiencies have been observed in many molecular simulations for various mixtures properties using these simple rules [22-25].

In engineering, the inadequacy of LB combining rules has been corrected using binary interaction parameters. The binary interaction parameters are often optimized using VLE calculations for binary mixtures and matching with experimental values [26]. The idea in this work is to circumvent or obscure the use of binary interaction parameters by employing more appropriate combining rules with theoretical basis.

In a word, the impetus in this work is improving the accuracy of cubic EoSs in describing the PVT of hydrocarbon systems. This study attempts to extend the comparisons made by Ahmed [11] to more EoSs. Further, this study evaluates theoretical combining rules. The subject of this work is furthermore extended to LNGs, some synthetic natural gases and some middle range hydrocarbon mixtures. This study would be comparative in nature. Van der Waals mixing rules are used for all EoSs. Ten combining rules available in literature are combined with seven well- known PVT relations to predict the densities of natural gas mixtures. The best pairs are introduced and reported. No binary interaction parameter would be used.

\section{Equations of State (EoS)}

Thirteen different EoSs and their variants were used in this study. The two-parameter Redlich-Kwong-Soave (RKS) [15] and their variants RKT [27] and RKSS [28] were used. The two-parameter Peng-Robinson (PR76) [9], Robinson and Peng (PR78) [29] and their variants PRT [30] and PRG [31] were used. The three-parameter Patel-Teja (PT) [4] and its variant by Valderrama (PTV) [5] were used. The threeparameter Schmidt and Wenzel (SW) [3], Guo and Du (GU) [6] and Mosen-Nia et al. (MMM) [14] were used. Salim and Trebble (TBS) [32] was a four-parameter EoS that was also used in this study. The PVT of the EoSs are provided in Figure 1.

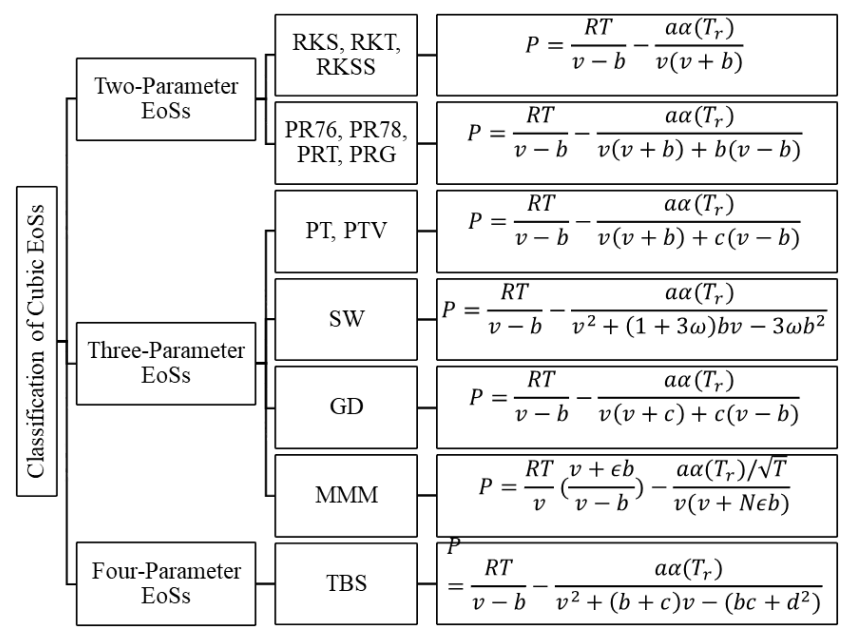

Figure 1. The PVT relationships for EoSs used in this study.

\section{Mixing Rules}

EoSs require mixing rules to describe mixture properties. For hydrocarbon systems, van der Waals mixing rules are often used:

$a=\sum_{i} \sum_{j} x_{i} x_{j} a_{i j}$

$a_{i j}=\sqrt{a_{i i} a_{j j}}\left(1-k_{i j}\right)$

$b=\sum_{i} \sum_{j} x_{i} x_{j} b_{i j}$

$w=\sum_{i} x_{j} w_{j}$

where $x$ is the mole fraction, a is the attractive parameter, $b$ is the co-volume parameter and $w$ can be $c$ or $d$. The parameter $k_{i j}$ is the binary interaction parameter, which is set to zero in this work. This parameter is used to correct attractive parameter combining rule expressed by Eq. (7). Eq. (5) is used for calculating $b_{\mathrm{ij}}$. Eq. (7) and Eq.(5) are the LB combining rules for EoS calculations.

Combining rules target the energy and the size parameters $a$ and $b$ in the van der Waals mixing rules (Eqs. 6 and 8). Eq. (9), which is a linear mixing rule, would be applied to $\mathrm{c}$ and $\mathrm{d}$ of the three-parameter and four-parameter EoSs. LB combining rules are the oldest and the most common ones used in practice but due to its inaccurate prediction of properties, other combining rules have also been proposed. Therefore, comparisons with different combining rules would be employed - that is, Lorentz- 
Berthelot (LB) [20, 21], Kohler (K) [33], Hudson and McCoubrey (HMC) [34], Fender-Halsey (FH) [35], Sikora (S) [36], Smith-Kong (SK) [37, 38], Halgren (HHG) [39], Waldman and Hagler (WH) [40] and Al-Matar and Rockstraw (M1 and M2) [41].

\subsection{Kohler (K)}

Kohler [33] developed his combining rule based on dispersion energy. He proposed the following expression for the energy parameter a:

$a_{i j}{ }^{K}=2^{7}\left[\frac{b_{i i} b_{j j}}{b_{i i}+b_{j j}}\right]^{6} \frac{\alpha_{i i} \alpha_{j j}}{\alpha_{j j}^{2} b_{i i}^{6} a_{i i}+\alpha_{i i}^{2} b_{j j}^{6} a_{j j}} a_{i i} a_{j j}$

The equation has a physical property called polarizability $\alpha$ which "quantifies the distortion of the overall charge distribution of an atom, group or molecule by an electric field." Values of polarizability of some components that commonly exist in petroleum fluids are presented in Table 1. Lorentz rule was proposed to be used for the repulsive parameter $b$ as given by Eq. (5).

Table 1. Polarizability and ionization potential values for some pure components used in this study [42].

\begin{tabular}{|c|c|c|}
\hline Component & $\boldsymbol{I}$ (Joule) & $\left.\boldsymbol{A} \mathbf{( 1 0}^{-\mathbf{2 4}} \mathbf{c m}^{\mathbf{3}}\right)$ \\
\hline $\mathrm{He}$ & $3.93934 \times 10^{-18}$ & 0.204956 \\
\hline $\mathrm{O}_{2}$ & $1.93378 \times 10^{-18}$ & 1.5812 \\
\hline $\mathrm{CO}_{2}$ & $2.20668 \times 10^{-18}$ & 2.911 \\
\hline $\mathrm{N}_{2}$ & $2.49632 \times 10^{-18}$ & 1.7403 \\
\hline $\mathrm{C}_{1}$ & $2.02034 \times 10^{-18}$ & 2.593 \\
\hline $\mathrm{C}_{2}$ & $1.85212 \times 10^{-18}$ & 4.47 \\
\hline $\mathrm{C}_{3}$ & $1.75438 \times 10^{-18}$ & 6.29 \\
\hline$i-\mathrm{C}_{4}$ & $1.6935 \times 10^{-18}$ & 8.14 \\
\hline$n-\mathrm{C}_{4}$ & $1.68709 \times 10^{-18}$ & 8.2 \\
\hline$i-\mathrm{C}_{5}$ & $1.65345 \times 10^{-18}$ & - \\
\hline$n-\mathrm{C}_{5}$ & $1.64704 \times 10^{-18}$ & 9.99 \\
\hline$n-\mathrm{C}_{6}$ & $1.623 \times 10^{-18}$ & 11.9 \\
\hline$n-\mathrm{C}_{7}$ & $1.59096 \times 10^{-18}$ & - \\
\hline Moluene & $1.41434 \times 10^{-18}$ & 11.8 \\
\hline$n-\mathrm{C}_{8}$ & $1.57013 \times 10^{-18}$ & 15.9 \\
\hline & &
\end{tabular}

\subsection{Hudson-McCoubrey (HMC)}

Hudson and McCoubrey [34] involved the ionization potential parameter I of the pure components and co-volume effects of the mixture in their proposed combining rule for the energy parameter a:

$a_{i j}{ }^{H M C}=\frac{2 \sqrt{I_{i i} I_{j j}}}{I_{i i}+I_{j j}}\left[\frac{2 \sqrt{b_{i i} b_{j j}}}{b_{i i}+b_{j j}}\right]^{6} \sqrt{a_{i i} a_{j j}}$

The ionization potential is defined as "the energy which is necessary to remove the outermost electron from an atom or molecule." Table 1 provides I values for some components. For the repulsive parameter b, Lorentz rule is used (Eq. (5)).

\subsection{Fender-Halsey (FH)}

Harmonic mean has been proposed by Fender and Halsey [35] for the energy parameter a:

$a_{i j}{ }^{F H}=\frac{2 a_{i i} a_{j j}}{a_{i i}+a_{j j}}$

Lorentz rule is applied for the repulsive parameter b.

\subsection{Sikora $(S)$}

In both the energy and the repulsive parameters in Sikora's combining rules [36], "repulsion" is considered via deformation energies of the electron clouds and resulting unsymmetric collision diameters, which occur at small distances between overlapping atoms or molecules. Accordingly, aij and bij read:

$a_{i j} S=2^{15} \frac{I_{i i} I_{j j}}{\left(I_{i i}+I_{j j}\right)^{2}} \frac{\sqrt{a_{i i} b_{i i}{ }^{12} a_{j j} b_{j j}{ }^{12}}}{\left[\left(a_{i i} b_{i i}{ }^{12}\right)^{\frac{1}{13}}+\left(a_{j j} b_{j j}{ }^{12}\right)^{\frac{1}{13}}\right]^{13}} \sqrt{a_{i i} a_{j j}}$

$b_{i j}{ }^{S}=2^{-\frac{13}{12}}\left(b_{i i^{\frac{12}{13}}}+b_{j j^{\frac{12}{13}}}\right)^{\frac{13}{12}}$

\subsection{Smith-Kong (SK)}

Smith [37] developed a combining rule considering the repulsive interaction of simple molecules. His combining rule includes the atomic distortion theory for repulsion (Eq. 16). Kong [38] used a same approach for the energy parameter 'a' (Eq. 15):

$a_{i j} S K=\frac{2^{13} a_{i i} b_{i i}{ }^{6} a_{j j} b_{j j}{ }^{6}}{\left[\left(a_{i i} b_{i i}{ }^{12}\right)^{\frac{1}{13}}+\left(a_{j j} b_{j j}{ }^{12}\right)^{\frac{1}{13}}\right]^{13}}$

$b_{i j} S K=\left\{\frac{\left[\left(a_{i i} b_{i i}{ }^{12}\right)^{\frac{1}{13}}+\left(a_{j j} b_{j j}{ }^{12}\right)^{\frac{1}{13}}\right]^{13}}{2^{13} \sqrt{a_{i i} b_{i i}{ }^{6} a_{j j} b_{j j}{ }^{6}}}\right\}^{\frac{1}{6}}$

\subsection{Halgren (HHG)}

Halgren [39] proposed cubic mean for the repulsive parameter $b$ after doing experiments on noble gas mixtures because it described the van der Waals minimum energy distances very well. For the energy parameter a, a combination of the geometric and harmonic mean was applied to yield good descriptions of the experimental noble gas values:

$a_{i j}{ }^{H H G}=\frac{4 a_{i i} a_{j j}}{\left(\sqrt{a_{i i}}+\sqrt{a_{j j}}\right)^{2}}$

$b_{i j}{ }^{H H G}=\frac{b_{i i}{ }^{3}+b_{j j}{ }^{3}}{b_{i i}{ }^{2}+b_{j j}{ }^{2}}$

\subsection{Waldman-Hagler (WH)}

Waldman and Hagler [40] proposed the combining rules for the energy parameter $\mathrm{a}$ and repulsive parameter $\mathrm{b}$ based on graphical analysis on noble gas mixtures:

$a_{i j}{ }^{W H}=\frac{2 b_{i i}{ }^{3} b_{j j}{ }^{3}}{b_{i i}{ }^{6}+b_{j j}{ }^{6}} \sqrt{a_{i i} a_{j j}}$

$b_{i j}{ }^{W H}=\left[\frac{b_{i i}{ }^{6}+b_{j j}{ }^{6}}{2}\right]^{\frac{1}{6}}$

\subsection{Al-Matar (M1 and M2)}

Al-Matar and Rockstraw [41] proposed two combining rules by applying mathematical methods on noble gas mixtures. M1 is the first combining rule, which was proposed by considering that the energy and repulsive parameters a and $b$ of the pure components are equally weighted: 


$$
a_{i j}{ }^{M 1}=\frac{3 b_{i i}{ }^{3} b_{j j}{ }^{3}}{\sum_{L=0}^{2}\left(\frac{0.25\left(b_{i i}{ }^{3}+b_{j j}{ }^{3}\right)^{2}}{b_{i i}{ }^{L} b_{j j}{ }^{L}}\right)^{\frac{6}{6-2 L}}} \sqrt{a_{i i} a_{j j}}
$$

$b_{i j}{ }^{M 1}=\frac{1}{3} \sum_{L=0}^{2}\left(\frac{0.25\left(b_{i i}{ }^{3}+b_{j j}{ }^{3}\right)^{2}}{b_{i i}{ }^{2} b_{j j}{ }^{L}}\right)^{\frac{1}{6-2 L}}$

In the second combining rule, (M2), weighting matrices are used and they are found by fitting them to noble gas mixtures. Weighting matrices account for uneven contributions of the energy and repulsive parameters $a$ and $b$ of the pure components to the unlike quantities:

$$
\begin{aligned}
& a_{i j}{ }^{M 2}=\frac{0.03995 a_{i i}+0.9564698845 \sqrt{a_{i i} a_{j j}}+0.00355 a_{j j}}{0.282 b_{i i}{ }^{6}+0.4732 b_{i i}{ }^{3} b_{j j}{ }^{3}+0.2448 b_{j j}{ }^{6}} b_{i i}{ }^{3} b_{j j}{ }^{3} \\
& b_{i j}{ }^{M 2}=\left(0.282 b_{i i}{ }^{6}+0.4732 b_{i i}{ }^{3} b_{j j}{ }^{3}+0.2448 b_{j j}{ }^{6}\right)^{\frac{1}{6}}
\end{aligned}
$$

\section{Results and Discussion}

A data bank comprised of two binary mixtures, one ternary mixture, five liquefied natural gas (LNG) mixtures and seven synthetic natural gas mixtures was collected. In Table 2 the number of points, the temperature, pressure and density ranges of the binary and ternary mixtures are given. The ranges for temperature are limited to the applications of the mixtures in gas industry. In Tables 3 and 4 the ranges and compositions of LNGs are provided, respectively. One can find the ranges and compositions of the seven synthetic natural gas mixtures in Tables 5 and 6 .

The combining rules were compared by their effect on predicting densities using the equations of state. The percent average absolute deviation (\%AAD) of the predicted densities from experimental values are expressed by:

$$
\% A A D=\frac{100}{N P} \sum_{i}^{N P} \frac{\left|\rho_{i}^{\exp }-\rho_{i}^{c a l}\right|}{\rho_{i}^{\exp }}
$$

Table 2. Binary and ternary mixtures used in this study [43].

\begin{tabular}{|c|c|c|c|c|}
\hline $\begin{array}{c}\text { Binary/Ternary } \\
\text { System }\end{array}$ & No. of data points & $\begin{array}{c}\text { Temperature Range } \\
(\mathrm{K})\end{array}$ & $\begin{array}{c}\text { Pressure Range } \\
(\mathrm{MPa})\end{array}$ & $\begin{array}{c}\text { Density Range } \\
\left(\mathrm{kg} / \mathrm{m}^{3}\right)\end{array}$ \\
\hline$n-\mathrm{C}_{5}+n-\mathrm{C}_{6}$ & 105 & $298.15-348.15$ & $0.1-40$ & $572.51-686.15$ \\
\hline$n-\mathrm{C}_{6}+n-\mathrm{C}_{7}$ & 90 & $298.15-348.15$ & $0.1-40$ & $610.77-708.48$ \\
\hline$n-\mathrm{C}_{5}+n-\mathrm{C}_{6}+n-\mathrm{C}_{7}$ & 120 & $298.15-348.15$ & $0.1-40$ & $583.77-702.63$ \\
\hline
\end{tabular}

Table 3. LNG mixtures used in this study [44].

\begin{tabular}{|c|c|c|c|c|}
\hline LNG Mixture & Data points & Temperature Range (K) & Pressure Range $(\mathrm{MPa})$ & Density Range $\left(\mathrm{kg} / \mathrm{m}^{3}\right)$ \\
\hline LNG 1 & 31 & $105-135$ & $0.47938-8.74337$ & $451.578-498.2$ \\
\hline LNG 2 & 35 & $105-135$ & $0.18628-8.90791$ & $436.468-484.597$ \\
\hline LNG 3 & 28 & $105-135$ & $0.18832-8.37252$ & $437.71-485.11$ \\
\hline LNG 4 & 28 & $105-135$ & $0.18805-8.65236$ & $436.262-484.274$ \\
\hline LNG 5 & 28 & $105-135$ & $0.18718-6.83937$ & $395.82-446.529$ \\
\hline
\end{tabular}

Table 4. Composition of the LNG mixtures [44].

\begin{tabular}{|c|c|c|c|c|c|}
\hline Component & LNG 1 & LNG 2 & LNG 3 & LNG 4 & LNG 5 \\
\hline $\mathrm{CO}_{2}$ & 0 & 0 & 0 & 0 & 0 \\
\hline $\mathrm{N}_{2}$ & 0.6953 & 0.3504 & 0.5854 & 0.3435 & 0.1933 \\
\hline $\mathrm{C}_{1}$ & 81.5626 & 87.8854 & 84.6362 & 87.9716 & 97.8898 \\
\hline $\mathrm{C}_{2}$ & 13.3744 & 7.2738 & 12.8 & 7.24 & 0.999 \\
\hline $\mathrm{C}_{3}$ & 3.6793 & 2.9257 & 1.499 & 2.9 & 0.4971 \\
\hline $\mathrm{i}-\mathrm{C}_{4}$ & 0 & 0 & 0.2199 & 0.6428 & 0.1771 \\
\hline $\mathrm{n}-\mathrm{C}_{4}$ & 0.6884 & 1.5647 & 0.2093 & 0.6917 & 0.2092 \\
\hline $\mathrm{i}-\mathrm{C}_{5}$ & 0 & 0 & 0.0201 & 0.11 & 0.0183 \\
\hline $\mathrm{n}-\mathrm{C}_{5}$ & 0 & 0 & 0.0301 & 0.1004 & 0.0162 \\
\hline
\end{tabular}

Table 5. Synthetic natural gases used in comparisons [45-47].

\begin{tabular}{|c|c|c|c|c|}
\hline Mixtures & Data points & Temperature Range $(\mathrm{K})$ & Pressure Range $(\mathrm{MPa})$ & Density Range $\left(\mathrm{kg} / \mathrm{m}^{3}\right)$ \\
\hline Mix 1 & 41 & $250-450$ & $0.586-37.3445$ & $3.739-328.877$ \\
\hline Mix 2 & 33 & $250-450$ & $0.596-36.749$ & $3.877-338.88$ \\
\hline Mix 3 & 30 & $270-340$ & $3.447-34.474$ & $23.264-298.051$ \\
\hline Mix 4 & 28 & $253.15-323.15$ & $0.992-15.008$ & $6.106-177.978$ \\
\hline Mix 5 & 28 & $253.15-323.15$ & $1-15.023$ & $6.746-211.811$ \\
\hline Mix 6 & 59 & $250-450$ & $10-65$ & $52.7-396.94$ \\
\hline Mix 7 & 59 & $250-450$ & $10-65$ & $50.35-377.03$ \\
\hline
\end{tabular}


Table 6. Composition of the synthetic natural gases [45-47].

\begin{tabular}{|c|c|c|c|c|c|c|c|}
\hline Component & Mix 1 & Mix 2 & Mix 3 & Mix 4 & Mix 5 & Mix 6 & Mix 7 \\
\hline $\mathrm{He}$ & 0 & 0 & 0 & 0 & 0.015 & 0 & 0 \\
\hline $\mathrm{O}_{2}$ & 0 & 0 & 0 & 0 & 0.011 & 0 & 0 \\
\hline $\mathrm{CO}_{2}$ & 1.707 & 0 & 0.403 & 0.066 & 0.647 & 2.331 & 0 \\
\hline $\mathrm{N}_{2}$ & 1.699 & 0 & 2.031 & 0.841 & 1.474 & 3.496 & 0 \\
\hline $\mathrm{C}_{1}$ & 89.99 & 90.001 & 90.991 & 98.352 & 90.362 & 84.99 & 90.26 \\
\hline $\mathrm{C}_{2}$ & 3.15 & 4.565 & 2.949 & 0.511 & 5.708 & 5.529 & 5.828 \\
\hline $\mathrm{C}_{3}$ & 1.583 & 2.243 & 1.513 & 0.153 & 1.124 & 2.008 & 2.106 \\
\hline$i-\mathrm{C}_{4}$ & 0.781 & 1.14 & 0.755 & 0.021 & 0.301 & 0.401 & 0.412 \\
\hline$n-\mathrm{C}_{4}$ & 0.790 & 1.151 & 0.755 & 0.031 & 0.169 & 0.585 & 0.641 \\
\hline$i-\mathrm{C}_{5}$ & 0.150 & 0.450 & 0.299 & 0.008 & 0.059 & 0.169 & 0.214 \\
\hline$n-\mathrm{C}_{5}$ & 0.150 & 0.450 & 0.304 & 0.011 & 0.029 & 0.147 & 0.162 \\
\hline$n-\mathrm{C}_{6}$ & 0 & 0 & 0 & 0.005 & 0.058 & 0 & 0 \\
\hline$n-\mathrm{C}_{7}$ & 0 & 0 & 0 & 0 & 0 & 0.102 & 0.111 \\
\hline Toluene & 0 & 0 & 0 & 0 & 0 & 0.09 & 0.110 \\
\hline$n-\mathrm{C}_{8}$ & 0 & 0 & 0 & 0.0003 & 0.008 & 0.152 & 0.161 \\
\hline
\end{tabular}

Table 7. \%AAD for the RK family EoSs in predicting the liquid density of the binary and ternary mixtures using different combining rules.

\begin{tabular}{|c|c|c|c|c|c|}
\hline \multicolumn{6}{|l|}{ RKS } \\
\hline$n-\mathrm{C}_{5}+n-\mathrm{C}_{6}$ & $\% A A D$ & $n-\mathrm{C}_{6}+n-\mathrm{C}_{7}$ & $\% A A D$ & $n-\mathrm{C}_{5}+n-\mathrm{C}_{6}+n-\mathrm{C}_{7}$ & $\% A A D$ \\
\hline LB & 8.58 & LB & 10.27 & LB & 9.59 \\
\hline $\mathrm{FH}$ & 8.69 & $\mathrm{FH}$ & 10.32 & $\mathrm{FH}$ & 9.79 \\
\hline HMC & 8.78 & HMC & 10.37 & HMC & 9.99 \\
\hline $\mathrm{S}$ & 9.05 & $\mathrm{~S}$ & 10.50 & $\mathrm{~S}$ & 10.53 \\
\hline M1 & 9.28 & SK & 10.66 & SK & 11.18 \\
\hline SK & 9.32 & M1 & 10.68 & M1 & 11.22 \\
\hline HHG & 9.35 & $\mathrm{HHG}$ & 10.72 & $\mathrm{HHG}$ & 11.30 \\
\hline M2 & 9.57 & M2 & 10.80 & M2 & 11.68 \\
\hline $\mathrm{K}$ & 9.87 & $\mathrm{~K}$ & 10.94 & $\mathrm{~K}$ & 12.08 \\
\hline WH & 10.55 & WH & 11.35 & WH & 13.58 \\
\hline \multicolumn{6}{|l|}{ RKT } \\
\hline$n-\mathrm{C}_{5}+n-\mathrm{C}_{6}$ & $\% A A D$ & $n-\mathrm{C}_{6}+n-\mathrm{C}_{7}$ & $\% A A D$ & $n-\mathrm{C}_{5}+n-\mathrm{C}_{6}+n-\mathrm{C}_{7}$ & $\% A A D$ \\
\hline LB & 8.60 & LB & 10.29 & LB & 9.58 \\
\hline FH & 8.71 & $\mathrm{FH}$ & 10.34 & $\mathrm{FH}$ & 9.80 \\
\hline HMC & 8.80 & HMC & 10.39 & HMC & 10.00 \\
\hline $\mathrm{S}$ & 9.07 & $\mathrm{~S}$ & 10.52 & $\mathrm{~S}$ & 10.55 \\
\hline M1 & 9.29 & SK & 10.69 & SK & 11.20 \\
\hline SK & 9.34 & M1 & 10.71 & M1 & 11.24 \\
\hline HHG & 9.37 & HHG & 10.74 & HHG & 11.32 \\
\hline M2 & 9.59 & M2 & 10.82 & M2 & 11.70 \\
\hline $\mathrm{K}$ & 9.89 & $\mathrm{~K}$ & 10.96 & $\mathrm{~K}$ & 12.10 \\
\hline WH & 10.56 & WH & 11.15 & WH & 13.60 \\
\hline \multicolumn{6}{|l|}{ RKSS } \\
\hline$n-\mathrm{C}_{5}+n-\mathrm{C}_{6}$ & $\% A A D$ & $n-\mathrm{C}_{6}+n-\mathrm{C}_{7}$ & $\% A A D$ & $n-\mathrm{C}_{5}+n-\mathrm{C}_{6}+n-\mathrm{C}_{7}$ & $\% A A D$ \\
\hline LB & 8.60 & LB & 10.29 & LB & 9.58 \\
\hline $\mathrm{FH}$ & 8.71 & $\mathrm{FH}$ & 10.35 & $\mathrm{FH}$ & 9.81 \\
\hline HMC & 8.80 & HMC & 10.39 & HMC & 10.00 \\
\hline$S$ & 8.83 & $\mathrm{~S}$ & 10.53 & $\mathrm{~S}$ & 10.56 \\
\hline M1 & 9.30 & SK & 10.69 & SK & 11.21 \\
\hline SK & 9.35 & M1 & 10.71 & M1 & 11.24 \\
\hline HHG & 9.37 & HHG & 10.74 & HHG & 11.33 \\
\hline M2 & 9.59 & M2 & 10.83 & M2 & 11.70 \\
\hline $\mathrm{K}$ & 9.89 & $\mathrm{~K}$ & 10.97 & $\mathrm{~K}$ & 12.10 \\
\hline WH & 10.57 & WH & 11.38 & WH & 13.60 \\
\hline
\end{tabular}




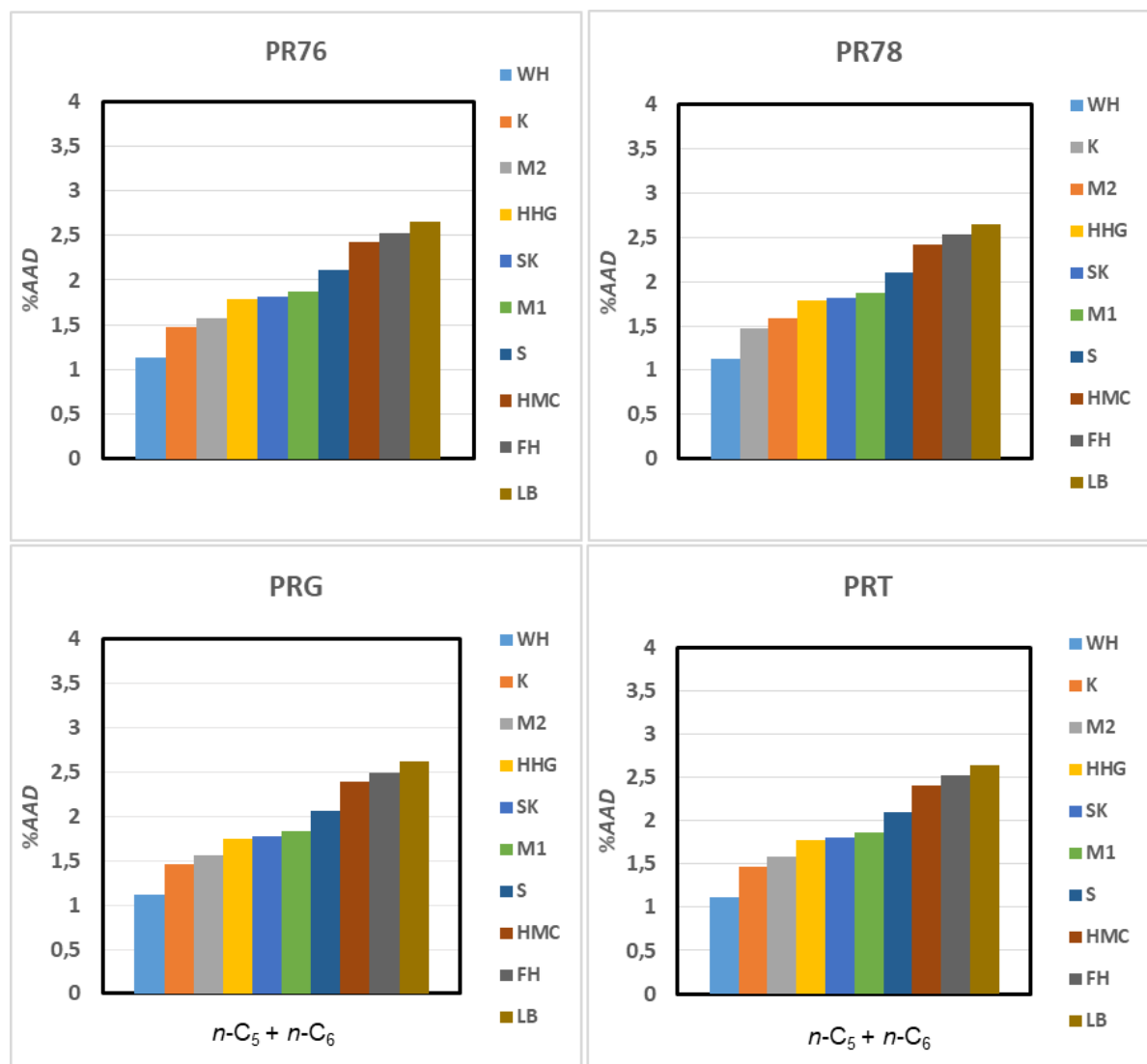

Figure 2. \%AAD of the PR Family EoSs in predicting the liquid densities of the $\mathrm{n}-C_{5}+\mathrm{n}-C_{6}$ binary mixtures using different combining rules.
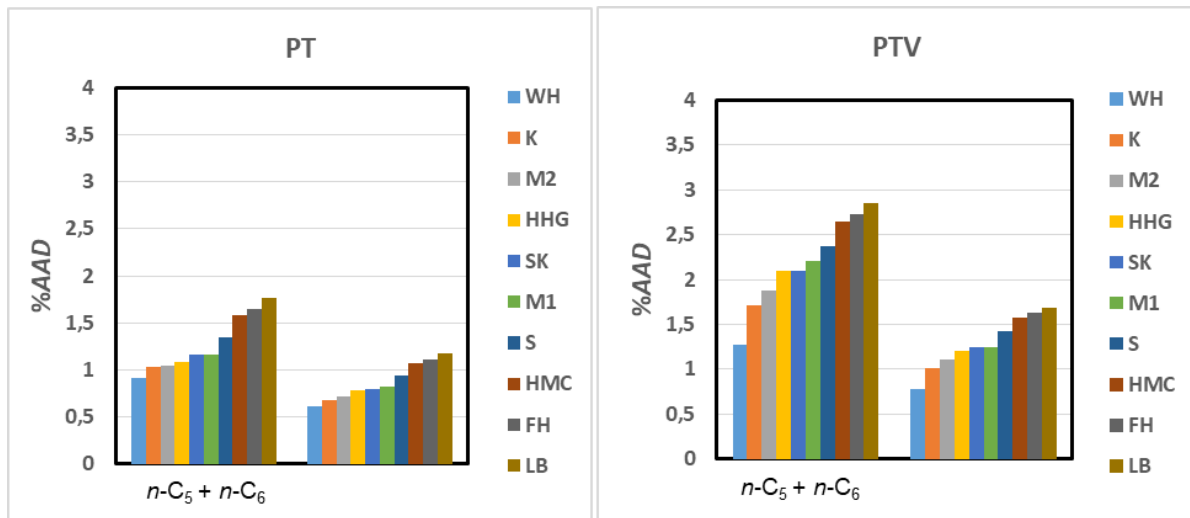

Figure 3. \%AAD of the PT and PTV EoSs in predicting the liquid densities of the binary mixtures $n-C_{5}+n-C_{6}$ and $n-C_{6}+n-$ $C_{7}$ using different combining rules.

Table 7 provides $\%$ AAD in predicting the liquid density of the binary/ternary systems using the RK family EoSs including RKS, RKT and RKSS. Results indicate that for these symmetric systems the LB combining rules are the best among the others when coupled with RK family EoSs. The next best combining rules were $\mathrm{FH}$ and HMC, respectively. The \%AAD was found to be around $9.6 \%$ for this family EoSs with LB combining rules.

When the combining rules were applied to the PR family EoSs in predicting the same liquid mixtures, the results were not coherent. Shown in Fig. 2, WH outperforms the other combining rules when used with the PR family EoSs in predicting the liquid density of $\mathrm{n}-\mathrm{C} 5+\mathrm{n}-\mathrm{C} 6$ mixtures. Clearly, the LB combining rules undermine the PR family EoSs among the others. For n-C6 + n-C7 mixtures, HHG was found the best and for the ternary mixture n-C5 + n-C6 + n$\mathrm{C} 7$, the combining rules $\mathrm{S}$ were found to be superior.
It was found that the three-parameter EoSs used in this work are more accurate than the two-parameter EoSs in predicting the densities of the liquid mixtures. One reason is that the EoSs are based on a mean field theory. They largely deviate in predicting liquid density near the critical point (reduced temperatures from 0.9 to 1 ). The ability of twoparameter EoS is often improved by incorporating a third parameter, which is normally a function of the true compressibility factor of fluids. In this way, the ability of the three-parameter EoS is partially augmented. The other reason is that the third parameter, which is similar to molar co-volume b, translates the EoS volume towards experimental value. In Fig. 3 one can see the accuracies of PT and PTV EoS with different combining rules. WH is the best combining rules. However, no particular conclusion can be drawn concerning the combining rules. Only the LB combining rules are not adequate for these EoSs when applied to the binary and ternary mixtures. 
Table 8. The best pairs of EoS/combining rules in predicting the liquid densities of the binary and ternary mixtures.

\begin{tabular}{|l|c|c|}
\hline EoS & Combining rules & $\% A A D$ \\
\hline RKS & LB & 9.45 \\
\hline RKT & LB & 9.45 \\
\hline RKSS & LB & 9.46 \\
\hline PR76 & HHG & $1.19(1.72)^{\text {a }}$ \\
\hline PR78 & HHG & $1.19(1.72)$ \\
\hline PRG & HHG & $1.18(1.70)$ \\
\hline PRT & HHG & $1.18(1.72)$ \\
\hline PT & HHG & $0.87(1.47)$ \\
\hline PTV & M2 & $1.36(2.22)$ \\
\hline SW & HMC & $0.65(0.7)$ \\
\hline GD & HHG & $0.82(1.37)$ \\
\hline MMM & WH & $5.58(8.29)$ \\
\hline TBS & LB & 2.13 \\
\hline
\end{tabular}

${ }^{\text {a }}$ The number in the parenthesis indicates the $\% A A D$ by LB combining rules

Given in Table 8 are the \%AADs in predicting the liquid densities of the binary and ternary mixtures for the best combinations of the EoSs with combining rules. Clearly, the LB combining rules are the best with the RK family EoSs (RKS, RKT, RKSS) and the TBS EoS. For the rest, the LB combining rules has no priority. Clearly, use of the other combining rules, in particular HHG greatly improves the prediction ability of PR family EoSs (PR76, PR78, PRT, PRG) for these binary and ternary mixtures.

Table 9. The best pairs of EoS/combining rules in predicting the liquid densities of the LNG mixtures.

\begin{tabular}{|l|c|c|}
\hline EoS & Combining rules & $\% A A D$ \\
\hline RKS & LB & 1.08 \\
\hline RKT & LB & 1.13 \\
\hline RKSS & LB & 1.12 \\
\hline PR76 & K & $2.27(11.48)^{\mathrm{a}}$ \\
\hline PR78 & K & $2.28(11.48)$ \\
\hline PRG & K & $2.26(11.62)$ \\
\hline PRT & K & $2.25(11.60)$ \\
\hline PT & K & $0.79(1.78)$ \\
\hline PTV & HHG & $1.82(5.48)$ \\
\hline SW & LB & 0.25 \\
\hline GD & FH & $0.72(2.50)$ \\
\hline MMM & LB & 2.63 \\
\hline TBS & LB & 1.69 \\
\hline
\end{tabular}

${ }^{\text {a }}$ The number in the parenthesis indicates the $\% A A D$ by $\mathrm{LB}$ combining rules

The ability of the best combing rules with the EoSs in predicting the densities of LNG mixtures are provided in Table 9. Again, the LB combining rules are the best with RK family and TBS EoSs. LB is also superior with the SW and MMM EoSs. For PR family, however, the $\mathrm{K}$ combining rules are the best. Compared to LB combing rules, the $\mathrm{K}$ combining rules improve greatly the ability of the PR family. While the \%AAD is about $2.3 \%$ for the PR family with $\mathrm{K}$ combining rules, use of $\mathrm{LB}$ deteriorates the \%AAD to $11.5 \%$. For the rest, other combining rules have privilege as shown in Table 9.
Table 10. The best pairs of EoS/combining rules for predicting the densities of the synthetic natural gas mixtures.

\begin{tabular}{|l|c|c|}
\hline EoS & Combining rules & $\% A A D$ \\
\hline RKS & LB & 1.95 \\
\hline RKT & M1 & $2.30(2.85)$ \\
\hline RKSS & M1 & $3.02(3.36)$ \\
\hline PR76 & HHG & $1.91(3.66)^{\mathrm{a}}$ \\
\hline PR78 & HHG & $1.93(3.50)$ \\
\hline PRG & HHG & $1.97(3.33)$ \\
\hline PRT & HHG & $2.00(3.48)$ \\
\hline PT & LB & 1.08 \\
\hline PTV & LB & 1.20 \\
\hline SW & LB & 1.11 \\
\hline GD & M1 & $1.57(1.68)$ \\
\hline MMM & M1 & $4.16(5.84)$ \\
\hline TBS & LB & 1.44 \\
\hline
\end{tabular}

a The number in the parenthesis indicates the $\% A A D$ by $\mathrm{LB}$ combining rules

Shown in Fig. 4, the four-parameter TBS EoS with LB combining rules predict the liquid densities of binary and ternary mixtures with less \%AAD. The FM and HMC closely follow the $\mathrm{LB}$ combining rules.

TBS

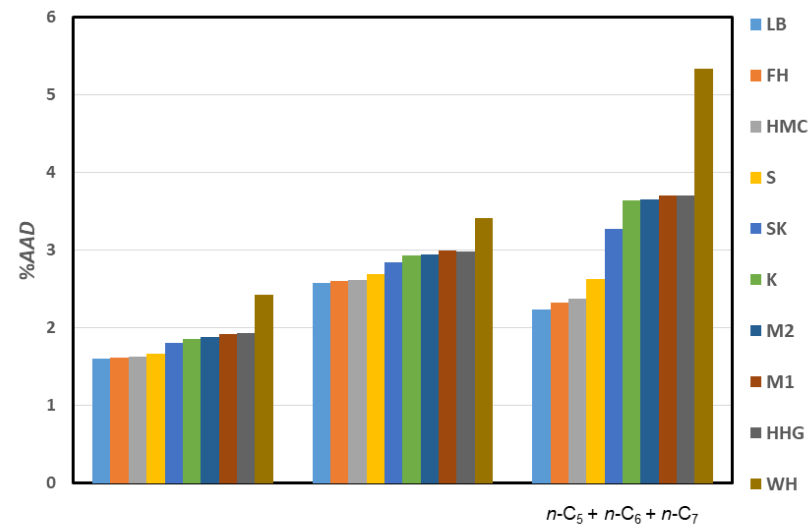

Figure 4. \%AAD of the TBS EOS in predicting the liquid densities of the binary mixtures $n-C_{5}+n-C_{6}$ and $n-C_{6}+n-C_{7}$ and the ternary mixture $n-C_{5}+n-C_{6}+n-C_{7}$ using different combining rules.

Comparisons made in Table 10 reveals that the LB and M1 are quite good for predicting the densities of synthetic natural gas mixtures for the most EoSs. Even when the M1 combining rules are the most accurate, $\mathrm{LB}$ combining rules are still close. Then, with the exception of PR family (PR76, PR78, PRT, PRG), LB combing rules are more appropriate for the other EoSs. For the PR family EoSs, however, HHG are more accurate.

Comparing Tables 8-10 makes it clear that for the RK family EoSs (RKS, RKT, RKSS), the SW and TBS equations of state, one can reliably use the LB combining rules. The PR family (PR76, PR78, PRG, PRT) are more accurate with HHG combining rules, however. Although for predicting the densities of LNG mixtures the $\mathrm{K}$ combining rules are the best, the use HHG provides an average \%AAD of circa $5 \%$. It means that one can rely on HHG mixing rules for PR family mixing rules. 


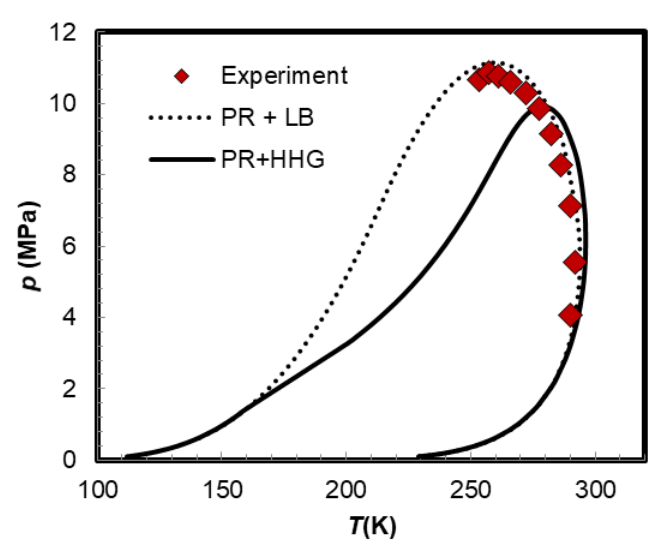

Figure 5. The phase envelope of the gas mixture $92.4 \% C_{1}+$ $3.21 \% C_{2}+0.71 \% C_{3}+0.70 \% n-C_{4}+0.64 \% n-C_{5}+0.71 \%$ $n-C_{6}+0.72 \% \mathrm{CO}_{2}+0.91 \% \mathrm{~N}_{2}$ using the PR EoS with $L B$ and $H H G$ combining rules (experimental data from $M u$ and Cui [48]).

Fig. 5 reveals that the LB combining rules with the PR EoS accurately calculates the phase envelope of the gas mixture $92.4 \% \mathrm{C} 1+3.21 \% \mathrm{C} 2+0.71 \% \mathrm{C} 3+0.70 \% \mathrm{n}-\mathrm{C} 4+$ $0.64 \%$ n-C5 $+0.71 \%$ n-C6 $+0.72 \% \mathrm{CO} 2+0.91 \% \mathrm{~N} 2$. However, the PR EoS with HHG combining rules are less accurate and deteriorate near the retrograde region. Nevertheless, one can still use HHG for calculating liquid density using the PR family, and the PR family EoS with LB combining rules for calculating vapor-liquid equilibria (VLE). It is worth noting that with the same properties needed for VLE calculation using the LB combining rules one can accurately calculates liquid density using the HHG combining rules. With the exception of SW, where LB combining rules are appropriate, the other three-parameter EoSs do not show compliance with any set of combing rules.

\section{Conclusions}

Thirteen EoSs have been coupled with ten combining rules in predicting the liquid and gas densities of two binaries, one ternary, five LNGs and seven synthetic natural gas mixtures. The comparisons revealed that for RK family EoSs (RKS, RKT, RKSS), the SW and TBS EoSs, the LB combing rules are the most accurate and should be preferred. For the PR family EoSs (PR76, PR78, PRT, PRG); however, it was found that HHG combining rules were the best.

\section{List of symbols}

$\begin{array}{ll}a & \text { Energy parameter in equation of state } \\ b & \text { Size parameter in equation of state } \\ I & \text { Ionization potential }(\mathrm{J}) \\ N P & \text { Number of points } \\ P & \text { Pressure }(\mathrm{MPa}) \\ r & \text { Distance } \\ R & \text { Universal gas constant }\left(8.314 \mathrm{~m}^{3} \mathrm{~Pa} \mathrm{~K}^{-1} \mathrm{~mol}^{-1}\right) \\ T & \text { Temperature }(\mathrm{K}) \\ v & \text { Molar volume }\left(\mathrm{m}^{3} \mathrm{kmol}^{-1}\right) \\ u & \text { Potential energy }(\mathrm{J})\end{array}$

\section{Abbreviations}

$A A D$ Average absolute deviation

EoS Equation of state

FH Fender-Halsey combining rule

GD Guo and Du equation of state

HHG Halgren combining rule

HMC Hudson-McCoubrey combining rule

$\mathrm{K} \quad$ Kohler combining rule
LB Lorentz-Berthelot combining rule

LNG Liquefied natural gas

M1 and M2 Al-Matar combining rules

MMM Mohsen-Nia-Modarress-Mansoori equation of state

PR Peng and Robinson equation of state

PRT Peng-Robinson-Twu equation of state

PRG Peng-Robinson-Gasem equation of state

PT Patel and Teja equation of state

PTV Patel-Teja-Valderrama equation of state

PVT Pressure-volume-temperature

RK Redlich and Kwong equation of state

RKS Redlich-Kwong-Soave equation of state

RKT Redlich-Kwong-Twu equation of state

RKSS Redlich-Kwong-Souahi-Sator equation of state

S Sikora combining rule

SK Smith-Kong combining rule

SW Schmidt and Wenzel equation of state

TBS Trebble-Bishnoi-Salim equation of state

WH Waldman-Hagler combining rule

\section{Greek letters}

$\alpha \quad$ Temperature-dependent term

$\sigma \quad$ Size parameter

$\varepsilon \quad$ Energy parameter

\section{Subscripts}

c Critical property

$i \quad$ Component number or index

LJ Lennard-Jones

$\mathrm{r} \quad$ Reduced property

\section{Acknowledgement}

M. S. Al-Harthy thanks to Sultan Qaboos University for providing MSc scholarship.

\section{References}

[1] K. Nasrifar, O. Bolland, M. Moshfeghian, "Predicting Natural Gas Dew Points from 15 Equations of State," Energy \& Fuels, 19, 561-572, 2005.

[2] O. Redlich, J. N. S. Kwong, "On the Thermodynamics of Solutions. V. An Equation of State. Fugacities of Gaseous Solutions," Chem. Rev., 44, 233-244, 1949.

[3] G. Schmidt, H. Wenzel, "A Modified Van der Waals Type Equation of State," Chem. Eng. Sci., 35, 15031512, 1980.

[4] N. C. Patel, A. S. Teja, "A New Cubic Equation of State for Fluids and Fluid Mixtures," Chem. Eng. Sci., 37, 463-473, 1982.

[5] J. O. Valderrama, "A Generalized Patel-Teja Equation of State for Polar and Nonpolar Fluids and their Mixtures," J. Chem. Eng. Jpn., 23, 87-91, 1990.

[6] T.-M. Guo, L. Du, "A Three-parameter Cubic Equation of State for Reservoir Fluids," Fluid Phase Equilib., 52, 47-57, 1989.

[7] T.-M. Guo, L. Du, “Application of the Du-Guo and SRK Equations of State to Predict the Phase Behavior of Chinese Reservoir Fluids," SPE Reservoir Eng., 379388, 1991.

[8] K. H. Coats, and G. T. Smart, "Application of a Regression-Based EoS PVT Program to Laboratory Data," SPE Reservoir Eng., 277-299, 1986.

[9] D. Y. Peng, D. B. Robinson, "A New Two-Constant Equation of State," Ind. Eng. Chem. Fundam., 5, 59-64, 1976. 
[10] D. Zudkevitch, J. Joffe, "Correlation and Prediction of Vapor-Liquid Equilibria with the Redlich-Kwong Equation of State," AIChE J., 16, 112-119, 1970.

[11] T. H. Ahmed, "Comparative Study of Eight Equations of State for Predicting Hydrocarbon Volumetric Phase Behavior," SPE Reservoir Engineering, 337-348, 1988.

[12] K. Nasrifar, O. Bolland, "Prediction of Thermodynamic Properties of Natural Gas Mixtures using 10 Equations of State including a New Cubic Two-constant Equation of State," J. Petrol. Sci. Eng., 51, 253-266, 2006.

[13] K. Nasrifar, M. Moshfeghian, "Application of an Improved Equation of State to Reservoir Fluids: Computation of Minimum Miscibility Pressure," J. Pet. Sci. Eng., 42, 223-234, 2004.

[14] M. Mohsen-Nia, H. Modarress, G. A. Mansoori, "A Cubic Hard-core Equation of State," Fluid Phase Equilib., 206, 27-39, 2003.

[15] G. Soave, "Equilibrium Constants from a Modified Redlich-Kwong Equation of State," Chem. Eng. Sci., 27, 1197-1203, 1972.

[16] J. M. Smith, H. C. Van Ness, M. M. Abbott, Introduction to Chemical Engineering Thermodynamics $7^{\text {th }}$ ed. McGraw-Hill: New York, NY, 2005.

[17] A. Pina-Martinez, Y. L. Guennec, R. Privat, J. N. Jaubert, P. M. Mathias, "Analysis of the Combinations of Property Data That Are Suitable for a Safe Estimation of Consistent Twu $\alpha$-Function Parameters: Updated Parameter Values for the Translated-Consistent $t c$-PR and $t c$-RK Cubic Equations of State," J. Chem. \& Eng. Data, 63, 3980-3988, 2018.

[18] W. Zhao, X. Sun, L. Xia, S. Xiang, "Research into Polynomial Alpha Function for the Cubic Equation of State," Ind. Eng. Chem. Research., 57, 12602-12623, 2018.

[19] F. Farshchi-Tabrizi, K. Nasrifar, "Application of Predictive Equations of State in Calculating Phase Envelopes and Critical Points," J. Nat. Gas Sci. Eng., 2, 21-28, 2010.

[20] H. A. Lorentz, "Ueber die Anwendung des Satzes vom Virial in der Kinetischen Theorie der Gase," Annalen der Physik, 12, 127-136, 1881.

[21] D. Berthelot, Comptes Rendus de l'Académie des Sciences Paris, 126, 1703-1706, 1889.

[22] C. Desgranges, J. Delhommelle, "Evaluation of the grand-canonical partition function using expanded Wang-Landau simulations. III. Impact of combining rules on mixtures properties," The Journal of Chemical Physics, 140, 104-109, 2014.

[23] D. Boda, and D. Henderson, "The effects of deviations from Lorentz-Berthelot rules on the properties of a simple mixture," Molecular Physics, 106, 2367-2370, 2008.

[24] W. Song, P. J. Rossky, M. Maroncelli, "Modeling alkane+perfluoroalkane interactions using all-atom potentials: Failure of the usual combining rules," The Journal of Chemical Physics, 119, 9145-9162, 2003.

[25] J. Delhommelle, P. Millie, "Inadequacy of the LorentzBerthelot combining rules for accurate predictions of equilibrium properties by molecular simulation," Molecular Physics, 99, 619-625, 2001.

[26] K. Nasrifar, M. Moshfeghian, "Vapor-Liquid Equilibria of Binary Associating Fluids Using a Cubic Equation of State with Limited Data," Ind. Eng. Chem. Res., 53, 2052-2061, 2014.
[27] C. H. Twu, J. E. Coon, J. R. Cunningham, “A New Generalized Alpha Function for a Cubic Equation of State Part 2. Redlich-Kwong Equation," Fluid Phase Equilib., 105, 61-69, 1995b.

[28] F. Souahi, S. Sator, S. A. Albne, F. K. Kies, C. E. Chitour, "Development of a New Form for the Alpha Function of the Redlich-Kwong Cubic Equation of State." Fluid Phase Equilib., 153, 73-80, 1998.

[29] D. B. Robinson, D. Y. Peng, "The characterization of the heptanes and heavier fractions for the GPA PengRobinson programs, Gas processors association, Research report RR-28," (Booklet only sold by the Gas Processors Association, GPA), 1978.

[30] C. H. Twu, J. E. Coon, J. R. Cunningham, “A New Generalized Alpha Function for a Cubic Equation of State Part 1. Peng-Robinson Equation," Fluid Phase Equilib., 105, 49-59, 1995a.

[31] K. A. M. Gasem, W. Gao, Z. Pan, R. L. Robinson, “A Modified Temperature Dependence for the PengRobinson Equation of State," Fluid Phase Equilib., 181, 113-125, 2001

[32] P. H. Salim, M. A. Trebble, “A Modified TrebbleBishnoi Equation of State: Thermodynamic Consistency Revisited," Fluid Phase Equilib., 65, 59-71, 1991.

[33] F. Kohler, "Zur berechnung der wechselwirkungsenergie zwischen ungleichen molekiilen in bin\&en fliissigen mischungen," Monatsh. Chem., 88, 857-877, 1957.

[34] G. H. Hudson, J. C. McCoubrey, "Intermolecular Forces between Unlike Molecules: A More Complete Form of Combining Rules," Transactions of the Faraday Society 56, 761-766, 1960.

[35] B. E. F. Fender, and G. D. Halsey, "Second Virial Coefficients of Argon, Krypton, and Argon- Krypton Mixtures at Low Temperatures," J. Chem. Phys. 3, 1881-1888, 1962.

[36] P. T. Sikora, "Combining Rules for Spherically Symmetric Intermolecular Potentials," J. Phys. B, 3, 1475-1482, 1970.

[37] F. T. Smith, "Atomic Distortion and Combining Rule for Repulsive Potentials," Phys. Rev. A, 5, 1708-1713, 1972.

[38] C. L. Kong, "Combining Rules for Intermolecular Potential Parameters. II. Rules for the Lenard-Jones Potential (6-12) and the Morse Potential," J. Chem. Phys., 59, 2464-2467, 1973.

[39] T. A. Halgren, "The Representation of van der Waals (vdW) Interactions in Molecular Mechanics Force Field: Potential Form, Combining Rules, and vdW parameters," J. Am. Chem. Soc., 114, 7827-7843, 1992.

[40] M. Waldman, A. T. Hagler, "New Combining Rules for Rare Gas van der Waals Parameters," J. Comput. Chem., 14, 1077-1084, 1993.

[41] A. K. Al-Matar, D. A. Rockstraw, "A generating Equation for Mixing Rules and Two New Mixing Rules for Interatomic Potential Energy Parameters," $J$. Comput. Chem., 25, 660-668, 2004.

[42] D. R. Lide, CRC Handbook of Chemistry and Physics, 84th edition. CRC Press, 2003-2004.

[43] D. Pečar, V. Doleček, "Isothermal Compressibilities and Isobaric Expansibilities of Pentane, Hexane, Heptane and their Binary and Ternary Mixtures from Density Measurements," Fluid Phase Equilib., 211, 109-127, 2003. 
[44] R. Lentner, M. Richter, R. Kleinrahm, R. Span, "Density Measurements of Liquefied Natural Gas (LNG) over the Temperature Range from (105 to 135) $\mathrm{K}$ at Pressures up to 8.9 MPa." J. Chem. Thermodyn., 112, 68-76, 2017.

[45] M. Atilhan, S. Aparicio, F. Karadas, K. R. Hall, R. Alcalde, "Isothermal P $\rho$ T Measurements on Qatar's North Field Type Synthetic Natural Gas Mixtures using a Vibrating-tube Densimeter," J. Chem. Thermodyn., $53,1-8,2012$.
[46] M. O. McLinden, "P- $\rho-T$ Behavior of Four Lean Synthetic Natural-Gas-Like Mixtures from $250 \mathrm{~K}$ to 450 K with Pressures to $37 \mathrm{MPa}$," J. Chem. Eng. Data, 56, 606-6013, 2011.

[47] P. Patil, S. Ejaz, M. Atilhan, D. Cristancho, J. C. Holste, K. R. Hall, "Accurate Density Measurements for a $91 \%$ Methane Natural Gas-like Mixture," J. Chem. Thermodyn., 39, 1157-1163, 2007.

[48] L. Mu, Q. Cui, "Measurement and Prediction of Hydrocarbon Dew Points of Synthetic Natural Gas Mixtures," J. Chem. \& Eng. Data, 63, 4226-4233, 2018. 